verse of the fundamental theorem of Minkowski, which asserts that any convex body in $n$-dimensional space, which is symmetrical about the origin of co-ordinates $O$, and has volume greater than $2^{n}$, contains a point other than $O$ of every lattice of determinant 1. Hlawka's theorem, in its most fundamental form, states that if any body (whether convex or not) has volume less than 1 , then there exists a lattice of determinant 1 such that no lattice point, except possibly $O$, lies in the body. Mr. Rogers gave a simple proof of this theorem, restricting himself to the two-dimensional case for simplicity. We are given a bounded region in the plane, of area $A$ less than 1. Let $p$ be a large prime, and consider the lattice of points $(x, y)$ with

$$
x=u / \sqrt{ } p, \quad y=(s u+p v) / \sqrt{ } p,
$$

where $s$ is fixed, and $u, v$ take all integral values. This lattice has determinant 1 . We give $s$ the values $0,1, \ldots, p-1$, and suppose that each of the lattices has a point other than $O$ in the region. It can be proved that these $p$ points are all different. On the other hand, the total number of points of all the lattices in the region is easily found to be asymptotically $p A$ for large $p$. Since $A<1$, this is impossible, and so one at least of the lattices satisfies the require. ment of having no point, except possibly $O$, in the region.

Dr. H. Heilbronn spoke about some recent joint work with Prof. Davenport on a problem to which they had been led by considerations' in the geometry of numbers. The problem was to find two lattice points in a given plane lattice, say $\left(X_{1}, Y_{1}\right)$ and $\left(X_{2}, Y_{2}\right)$, which generate the lattice, and for which $X_{1} Y_{2}$ is numerically small. Two such points would be, in a certain sense, each near one of the two coordinate axes. Expressed analytically, the problem becomes that of investigating the minimum of a bilinear form :

$$
B=(\alpha x+\beta y)(\gamma z+\delta t),
$$

when $x, y, z, t$ take all integral values which satisfy the condition $x t-y z= \pm 1$. The results are as follows ${ }^{2}$. If $\Delta=|\alpha \delta-\beta \gamma|$, one can always satisfy $|B| \leqslant 2 \Delta /(5+3 \sqrt{5})$. If certain special forms are excluded (namely, those equivalent to $(x+\theta y)(z+$ $\left.\theta^{\prime} t\right)$, where $\theta$ and $\theta^{\prime}$ are $\left.\frac{1}{2}(-1 \pm \sqrt{ } 5)\right)$, this can be improved to $|B| \leqslant \Delta /(4+2 \sqrt{2})$. If more special forms are excluded, this can be further improved to $|B| \leqslant \Delta /(3+3 \sqrt{ } 2)$. But this inequality cannot be further improved by similar exclusions. There is a striking contrast with Markoff's results on the minimum of an indefinite quadratic form $(\alpha x+\beta y)$ $(\gamma x+\delta y)$. There Markoff established the existence of an infinite sequence of possible minima. In the present problem, the first two possible minima of the bilinear form correspond to those of a quadratic form, but the third does not.

Prof. Davenport gave the third talk, which was on another joint paper ${ }^{3}$ with Dr. Heilbronn. Let $Q\left(u_{1}, \ldots, u_{r}\right)$ be an indefinite quadratic form in $r$ variables. It has been conjectured for many years that if $r \geqslant 5$, such a form takes arbitrarily small values for an infinity of integral values of the variables. If the form has rational coefficients, this is true, since it then represents zero infinitely often. The general conjecture has an interpretation in the geometry of numbers. It is that the region of $r$ dimensional space defined by

$$
\left|x_{1}^{2}-x_{2}^{2} \pm x_{8}^{2} \pm \ldots \pm x_{r}^{2}\right|<1,
$$

where $r \geqslant 5$, contains a point other than $O$ of every lattice. In the language of Mahler, this region is of 'infinite type'.

A special case of the conjecture was proved by Chowla in 1934, namely, when $Q=\lambda_{1} u_{1}{ }^{2}+\ldots$ $+{ }_{r} u_{r}{ }^{2}$ and when $r \geqslant 9$. In the present work the result is proved for this particular form when $r \geqslant 5$. The proof uses a modification of the powerful methods created by Hardy and Littlewood for the treatment of problems in additive number-theory.

Prof. L. J. Mordell expressed appreciation of the talks, and said that the work of Mr. Rogers was particularly interesting in view of the complication of Hlawka's original proof, and of the deep ideas associated with the theorem by Siegel, in his recent proof in the Annals of Mathematics. Mr. Rogers had shown the value of a new and elementary approach to an apparently difficult theorem.

${ }^{1}$ Ann. Math., in the press.

2 quart. J. Math., in the press.

${ }^{3} J$. London Math. Soc., in the press.

\section{COMPLETE DOCUMENTATION*}

$$
\text { BY DR. S. C. BRADFORD }
$$

$T$ HIS Conference is important historically, because it marks the beginning of a second stage in the progress of documentation. In the past, our main endeavour has been directed to bring about the collaboration of documentary agencies in the single task of making accessible the scattered records of thought and achievement, especially in science and technology, until to-day more decimally classified references are produced than different papers abstracted. But, as the result of statistical investigations, made in the main by the British National Section, we have come to see that this alone will not suffice to produce a tolerably complete index to the records of human endeavour. Half the records are overlooked by the existing abstracting and indexing agencies. We need a means of covering all this literature. The means has been discovered. Its promotion has commenced, and this begins a second stage in our activities.

Statistical analyses have been made of the whole intake of the Science Library, London, not merely of three per cent samples. The results are for that reason the more reliable, although there is still room for more research, for which financial aid would be desirable.

The investigations were divided into four parts : (i) It was established that about million useful scientific and technical papers are recorded every year in an aggregate of some 15,000 useful scientific periodicals, while 300 abstracting and indexing periodicals publish also $\frac{8}{4}$ million abstracts, or references. But these abstracts, or references, relate to only $\frac{1}{2}$ million different papers. So that more than half the useful discoveries and inventions are recorded, only to be buried on the library shelves.

(ii) This general analysis was confirmed by the examination of the literature of certain definite subjects. The details of the examination of electrical engineering literature show that only 11,500 different papers on this subject, out of an annual output of

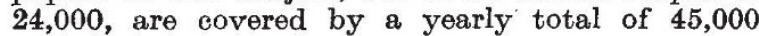

* Synopsis of a paper read at the sixteenth International Conference of the International Federation for Documentation at Paris, November 4-9, 1946 . 
abstracts, or references. These tests indicated also the basic reasons for this imperfection, which are : (a) individual abstracting agencies concentrate upon the periodicals devoted to their special subjects, together with a few of those the fields of which are closely allied to these subjects, and thus they miss the articles on their special subjects which occur in other periodicals the scopes of which are less closely related to them; and (b) abstracting agencies often ignore books, pamphlets, separate reports and patent specifications.

(iii) The actual scattering of articles on a given subject throughout the mass of periodical literature was investigated. This scattering is the necessary consequence of the fact that every branch of science is related in some degree to every other branch of science, so that a periodical on any branch of science may contain, from time to time, a paper which is of interest from the point of view of any other science. The actual law of scattering has been deduced, both from theoretical consideration, and from investigation of the actual articles indexed on a given subject. This law shows that it is practically impossible for an abstracting agency, acting alone, to pick up more than about half the articles published on its special subject.

(iv) The actual occurrence of these scattered articles, in the estimated quantity, was proved by a bulk experiment with the collaboration of the abstractors of a large abstracting bureau.

These experiments show conclusively that, somehow, every useful article in every useful periodical must be dealt with as it appears. There are two different ways of accomplishing this task. Either we could create a new organisation to index, or to abstract, each useful article in each useful periodical, independently of the subject of the article; or the useful periodicals could be divided into groups, according to the subjects treated by existing abstracting agencies, and these agencies should undertake to pass on the articles not within their scopes to the agencies who should be interested in their subjects.

The former method would be utterly uneconomic, because it would duplicate, without any adequate reason, the work of the existing skilled and experienced abstracting bureaux. The latter method needs much less additional expense. No interference is involved with the internal machinery of any abstracting agency. Each can continue to deal with and abstract as many periodicals as it desires and to publish its abstracts in any format, provided only that it passes on the articles not within its own scope.

The application of this principle has been commenced in Great Britain. A committee of important abstracting agencies has been formed, which has accepted the principle, and an agreement has been made by two of the most important agencies, British Abstracts and Science Abstracts, to work together so far as possible in this way. Bibliography is unforgettably indebted to Dr. J. A. Wilcken, secretary of the British Committee, for his outstanding services in this work.

The comprehensive indexing of books and other separate publications is essentially a matter for librarians and can best be achieved by improving the accessions lists of the national libraries of each country. In this way we can certainly achieve complete documentation.

[This plan was adopted unanimously by the Council of the International Federation for Documentation, and a small international committee was appointed to promote it.]

\section{SCIENCE MASTERS' ASSOCIATION}

\section{ANNUAL MEETING}

$T$

THE forty-fourth annual meeting of the Science Masters' Association was held at the Imperial College of Science and Technology, London, during January 1-3; and once again, in spite of the fact that the previous meeting was held only last Easter, the attendance was large, and an excellent programme of lectures and visits to works marked another successful gathering. There was also the usual exhibition of books and apparatus by the leading publishers and manufacturers of scientific apparatus, and the customary exhibit of apparatus devised and constructed by members themselves, and also one of graphic material of use in teaching. The Association has now returned to its old practice of holding its annual meeting during the Christmas holiday.

Giving the presidential address, Lord Horder spoke on the "Place of Science in Teaching", approaching this in the free and interesting spirit of one who saw things in their wider setting, and presenting some sound advice to his audience. His address, which sparkled at times with some keen wit and was illustrated by apposite anecdote, took three aspects of the subject: the pupil, the matter taught, and the teacher. The most important aspect is the pupil, who is still, it must be emphasized, a child, still half-savage, with recurring moods of self-exhibitionism and fantasy which can last well into undergraduate life and which must neither be unduly encouraged nor neglected. The task of the teacher is to help the child on the road to the full development of personality; he cannot create such personality.

The matter taught must be free from domination by the examination spectre which stultifies curiosity and impairs receptive power, and must avoid undue emphasis on application at the expense of first principles. Through the study of science the youthful mind can acquire habits of clear thinking and direct expression which lead it to become a joy to itself and a source of benefit to others.

The attitude of the teacher is of fundamental importance to the efficiency of his teaching. This attitude must be animated by two ideals : first, that science must be free and self-contained, owing subservience to neither Church, State nor the pressure of economic adjustment; secondly, the discoveries of science must be used for the benefit of humanity. The present troubles of the world are due to too little science, not too much. To drive science underground by the cessation of research would be to repeat all the evils of the Dark Ages. What is important is that we retain sufficient control to drive the machine and not allow it to drive us. Upon these ideals it is important that science teachers should stand firm. The chief aim of science is truth, and truth grows as does a living plant, which suffers as much from forcing as it does from neglect.

The Association was again fortunate in having lectures from men of science of eminence in their own fields. Mr. E. J. Bowen spoke on "Elementary WaveMechanics", presenting a treatment which opened the way to deriving ideas of great value in chemistry teaching; Sir Edward Salisbury discussed "The Changing Flora of Britain", describing the nature of large-scale changes since the Glacial period; Sir George Thomson spoke on "Atomic Energy in Peace and War", ending with a stimulating consideration of present problems; Prof. J. Z. Young took the 